\title{
Einführung zum Thema
}

Diabetologe 2016 $\cdot 12: 4-5$

DOI 10.1007/s11428-015-0058-1

Online publiziert: 29. Januar 2016

๑) Springer-Verlag Berlin Heidelberg 2016

CrossMark

Im Jahr 2014 erblickten in Deutschland 715.000 Kinder das Licht der Welt. In 32.000 Fällen wurde bei der Mutter die Diagnose Gestationsdiabetes (GDM) gestellt, das entspricht 4,47 \% aller Schwangerschaften. Die GDM-Häufigkeit verdreifachte sich in den letzten 10 Jahren, mit weiter steigender Tendenz. Hinzu kommen rund 6400 Schwangerschaften bei Frauen, deren Typ-1- oder Typ-2Diabetes bereits vor der Gravidität bekannt war, entsprechend 0,92 \% aller Geburten. Dieser Prozentsatz ist seit einigen Jahren konstant. Der Anteil schwangerer Frauen mit Typ-2-Diabetes an dieser Gruppe ist parallel zur steigenden Rate von Adipositas schwangerer Frauen gewachsen und liegt schätzungsweise in Zentren bei 20-40 \%. Eine Geburtsklinik mit 1000 Geburten pro Jahr wird somit im Mittel 54-mal innerhalb von 12 Monaten mit den Anforderungen für die Betreuung einer diabetischen Schwangerschaft konfrontiert. Das Thema Diabetes ist damit zum ständigen Geburtsbegleiter geworden.

\section{》) Schwangere mit Diabetes sind keine Seltenheit mehr}

Die epidemischen Ausmaße von Übergewicht und Fettleibigkeit in der Bevölkerung haben längst die jüngeren Jahrgänge und damit die reproduktiven Jahre der Frauen erreicht, was zu einer $\mathrm{Zu}$ nahme der Fälle von Gestationsdiabetes und Schwangeren mit Typ-2-Diabetes führt. Übergewicht und Adipositas steigern aber bereits an sich - auch ohne Diabetes - die Risiken für Mutter und Kind, worauf die Frauenärztin und Diabetologin Ute Schäfer-Graf in ihrer Übersicht eingeht. So kommt es bei einem prägraviden BMI (Body-Mass-In-

\author{
H. Kleinwechter ${ }^{1}$ W. A. Scherbaum ${ }^{2}$ \\ 1 Diabetes-Schwerpunktpraxis und Schulungszentrum, diabetologikum kiel, Kiel, Deutschland \\ ${ }^{2}$ Universitätsklinikum Düsseldorf, Heinrich-Heine-Universität Düsseldorf, Düsseldorf, Deutschland
}

\section{Diabetes und Schwangerschaft}

dex) von $\geq 30 \mathrm{~kg} / \mathrm{m}^{2}$ zu einer Verdoppelung des Fehlbildungsrisikos. Ab einem BMI von $35 \mathrm{~kg} / \mathrm{m}^{2}$ kann der Untersucher nur unter Einsatz starker körperlicher Kräfte eine aussagefähige fetale Ultraschalldiagnostik durchführen, allerdings auch dann nur mit eingeschränkter Aussagekraft, denn Schallwellen werden von Fettschichten absorbiert.

Für die Empfehlung, den Nachwuchs möglichst lange zu stillen, ist glücklicherweise kein Votum einer Ethikkommission, auch keine Zulassung durch die europäische Arzneimittelbehörde erforderlich. Im Rahmen einer diabetischen Schwangerschaft hat das Stillen einen besonderen Stellenwert. Es erfordert jedoch besondere Anstrengungen, den diabetischen Schwangeren das Stillen nahezulegen, sonst wird der Versuch, den Säugling an der mütterlichen Brust zu ernähren, allzu oft scheitern. Den derzeitigen Stand des Wissens und die vielen Vorteile des Stillens erläutert Jens Stupin, ein auf diesem Gebiet sehr erfahrener und engagierter Frauenarzt. Die vielen positiven Seiten des Stillens für Mütter und Kinder sprechen für sich. Stillen über einen Zeitraum von 3 oder mehr Monaten ist für die Mütter nach einem GDM als Diabetesprävention anzusehen.

\section{I) Bei Schwangeren mit Diabetes stellen sich besondere rechtliche Fragen}

Rechtsanwalt Oliver Ebert, Vorsitzender des Ausschusses Soziales der DDG (Deutsche Diabetes-Gesellschaft), klärt darüber auf, wie lange ein Beschäftigungsverbot bei Schwangeren dauert, wann es ausgesprochen werden kann und wer das verfügen darf, ebenso über weitere Rechtsfragen, wie die Verord- nungsmenge von Blutzuckerteststreifen und warum einer Schwangeren meist problemlos eine Insulinpumpe verordnet werden kann. Falls eine junge Mutter nach einem Gestationsdiabetes den Nachsorgetermin 2 Monate nach der Geburt versäumt, stellt sich die Frage, ob Sie einfach bei ihr anrufen lassen und einen Termin vergeben können. Hätten Sie gewusst, dass Sie dann u. U. mit dem Patientenrechtegesetz und dem Wettbewerbsrecht in Kollision geraten? Ein immer häufiger auftretendes Szenario ist Folgendes: Eine Migrantin mit Gestationsdiabetes, die allein in die Sprechstunde gekommen ist und kein Deutsch versteht, benötigt Insulin. Was von Ihrer Aufklärung der Schwangeren gegenüber dazu verlangt wird und ob Sie mit der Verordnung von Insulin vielleicht noch warten sollten, erfahren Sie ebenfalls im Artikel über die Rechtsfragen.

$\mathrm{Zu}$ den wichtigsten Risiken von Neugeborenen diabetischer Mütter zählen Hypoglykämien. Neugeborene können aber nicht darauf hinweisen, dass sie Heißhunger haben, schwitzen oder zittrig sind. Somit stellt sich die Frage, wie Hypoglykämien bei Neugeborenen diagnostiziert werden und ob diese Diagnose überall einheitlichen Konsens findet. Darüber berichtet der Neonatologe Christoph Bührer. Auch Antworten auf die Fragen, ob Blutzuckerselbstmessgeräte im Kreißsaal für die Diagnostik geeignet sind oder nicht, welche akuten Auswirkungen und Langzeitfolgen Hypoglykämien bei den soeben geborenen Kindern haben können, welche Therapien angemessen sind und was Diabetologen bereits zur Prävention beitragen können, finden sich in seinem Beitrag. Auch das bereits besprochene 
Stillen wird hier in seiner präventiven Dimension erweitert.

Im Jahr 1989 wurde erstmals in der vielzitierten St. Vincent-Deklaration von IDF („International Diabetes Federation“) und WHO („World Health Organization") Europa gefordert, Verlauf und Ausgang diabetischer Schwangerschaften an die Ergebnisse von Müttern ohne Diabetes anzugleichen. Leider ist dieses hochgesteckte Ziel auch nach mehr als 25 Jahren noch eine Illusion. Zum einen liegt dies daran, dass in Deutschland höchstens $50 \%$ der Patienten mit Typ-1-Diabetes in das DMP („disease management program") eingeschrieben sind und darauf vertrauen dürfen, leitliniengerecht behandelt zu werden. Die verbleibenden Patienten sind viel $\mathrm{zu}$ häufig sich selbst überlassen, sprechen teils 1 Jahr lang bei keinem Arzt vor, nutzen den hausärztlichen Sprechstundentresen als Insulinquelle und können sich nicht aufraffen, den nächst erreichbaren Diabetologen aufzusuchen. Zum anderen könnten Risiken für Mütter und Kinder sofort und effektiv durch eine sachgerechte und fundierte präkonzeptionelle Beratung abgemildert werden, was für Typ-1- und Typ-2-Diabetes gleichermaßen gilt. Eine Beratung vor der Schwangerschaft erhalten aber weniger als die Hälfte der Frauen. Die internistischen Diabetologen Norbert Demandt und Helmut Kleinwechter betonen in ihrer Übersicht, dass es bei der präkonzeptionellen Beratung entscheidend ist, sich auf das Wesentliche zu konzentrieren, mit einfachen Worten und klaren Empfehlungen effektiv zu sein. Ebenso wichtig ist, das Thema Schwangerschaft bei diabetischen Frauen im reproduktiven Alter immer wieder anzusprechen.

\section{》) Diabetische Frauen mit Kinderwunsch sollten präkonzeptionell beraten werden}

Die Betreuung von Schwangeren mit Diabetes umfasst im Vergleich zum von der Volkskrankheit Diabetes betroffenen Personenkreis insgesamt nur eine kleine Zahl. Auf diesen Patientenkreis aber sollten Diabetologen gut vorbereitet, d. h. ausgebildet sein und über hinreichend Erfahrungen verfügen. Es geht um unseren Nachwuchs und damit um unsere Zukunft. Es würde uns freuen, wenn dieses Schwerpunktheft von Der Diabetologe dazu beitragen kann, dass Diabetologen dieses anspruchsvolle Thema besser gestalten und ärztlich verantworten können.

\section{helmok heriweshte}

H. Kleinwechter

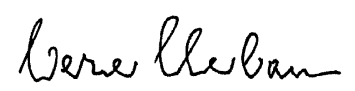

W. A. Scherbaum

\section{Korrespondenzadresse}

Dr. H. Kleinwechter
Diabetes-Schwerpunktpraxis
und Schulungszentrum,
diabetologikum kiel
Alter Markt 11, 24103 Kiel,
Deutschland
arzt@diabetologikum-kiel.de

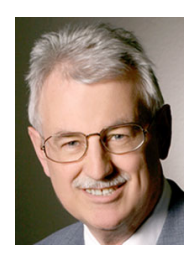

Univ.-Prof. (em.) Dr.

W. A. Scherbaum

Universitätsklinikum

Düsseldorf, Heinrich-Heine-

Universität Düsseldorf

Moorenstraße 5, 40225 Düsseldorf, Deutschland scherbaum@uniduesseldorf.de

Interessenkonflikt. H. Kleinwechter hat Vortragshonorare von NovoNordisk, BerlinChemie und Roche Diagnostics sowie Vortrags- und Beraterhonorare von Bayer Vital erhalten.

W.A. Scherbaum hat Vortrags- bzw. Beraterhonorare erhalten von: CompuGroup Medical, Roche Diagnostics, Amgen, Novartis, Sanofi, Servier, Lilly, AstraZeneca, Boehringer Ingelheim, MedUpdate.
Hier steht eine Anzeige. Springer 